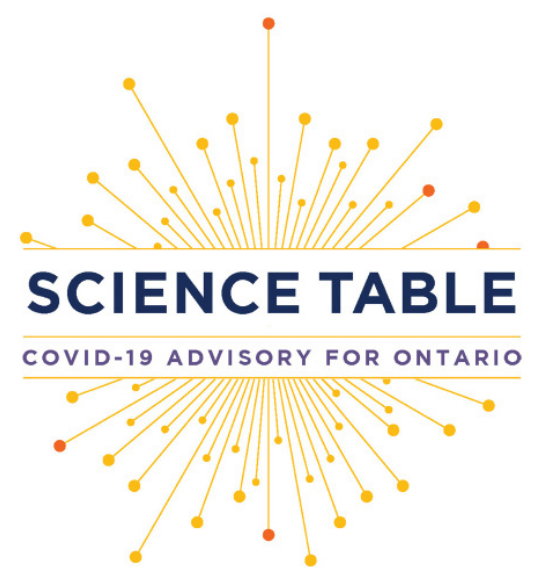

Version: $\mathbf{1 . 0}$

Published: October 7, 2021

Citation: Maunder RG, Heeney ND, Strudwick $\mathrm{G}$, et al. Burnout in hospital-based healthcare workers during COVID-19. Science Briefs of the Ontario COVID-19 Science Advisory Table. 2021;2(46). https://doi.org/10.47326/ ocsat.2021.02.46.1.0

Author Affiliations: The affiliations of the members of the Ontario COVID-19 Science Advisory Table can be found at https:// covid19-sciencetable.ca/.

Declarations of Interest: The declarations of interest of the members of the Ontario COVID-19 Science Advisory Table, its Working Groups, or its partners can be found at https:// covid19-sciencetable.ca/. The declarations of interest of external authors can be found under additional resources at https://doi. org/10.47326/ocsat.2021.02.46.1.0

About Us: The Ontario COVID-19 Science Advisory Table is a group of scientific experts and health system leaders who evaluate and report on emerging evidence relevant to the COVID-19 pandemic, to inform Ontario's response. Our mandate is to provide weekly summaries of relevant scientific evidence for the COVID-19 Health Coordination Table of the Province of Ontario, integrating information from existing scientific tables, Ontario's universities and agencies, and the best global evidence. The Science Table summarizes its findings for the Health Coordination Table and the public in Science Briefs.

The Mental Health Working Group comprises scientific experts and public health leaders with specific expertise in mental health. Their expertise spans mental health of children and youth, adults and geriatric populations, mental health of health care providers, women's health, mental health among LGBTQ persons, mental health among Black, Indigenous, and other racialized populations, and COVID-19. The Working Group evaluates emerging scientific evidence related to maintaining mental health during COVID-19, the mental health burden of disease and public health interventions on individuals across the lifespan, including children and adolescents, and the older adult population, as well as the need for assessment and recommendations
SCIENCE BRIEFS

\section{Burnout in Hospital-Based Healthcare Workers during COVID-19}

Robert G. Maunder, Natalie D. Heeney, Gillian Strudwick, Hwayeon Danielle Shin, Braden O'Neill, Nancy Young, Lianne P. Jeffs, Kali Barrett, Nicolas S. Bodmer, Karen B. Born, Jessica Hopkins, Peter Jüni, Anna Perkhun, David J. Price, Fahad Razak, Christopher J. Mushquash, Linda Mah on behalf of the Ontario COVID-19 Science Advisory Table and Mental Health Working Group

\section{Key Message}

Burnout is an occupational hazard in healthcare, which harms the healthcare system, patients, and healthcare workers. In the COVID-19 pandemic, burnout has increased to levels that pose a threat to maintaining a functioning healthcare workforce. Elevated burnout and other indicators of stress are anticipated to persist long after the pandemic.

The COVID-19 pandemic has created a cycle of understaffing alongside difficult work conditions which can drive burnout. Robust interventions to bolster individuals, improve work environments and address health system drivers of burnout are important to maintain and support hospital-based healthcare workers. Interventions need to target those most at risk and affected by burnout: nurses, intensive care unit and emergency department staff, women, recent graduates and trainees.

Interventions to reduce burnout need to be implemented at organizational and structural level of healthcare systems, complemented by intervention at the individual level. Further, leadership is a vital enabler to address burnout from organizational leaders and managers as well as policymakers.

Organizations need to ensure adequate staffing through ongoing evaluation of workload including mitigation of data entry and administrative burdens, efforts to reduce overtime and avoid long shifts, and staff deployment in areas where they lack training.

Approaches to mitigate, reduce and address burnout should be multi-faceted and include interventions to improve workplace conditions by fostering a supportive culture, relationships and leadership, as well as individual-level interventions (e.g., education, stress reduction tools, access support for moral distress).

\section{Summary}

\section{Background}

Burnout is characterized by emotional exhaustion, depersonalization, and diminished professional achievement. Prior to the COVID-19 pandemic, severe burnout was typically found in $20 \%-40 \%$ of healthcare workers. Contributors include workplace factors (e.g., workload, interpersonal conflict, moral distress, administrative burdens and documentation) and provider factors (e.g., low self-efficacy, emotional exhaustion, reduced sense of personal accomplishment,). Burnout is harmful for the healthcare system, workers, and patients. Risk factors have been exacerbated during the pandemic, resulting in an urgent need for intervention.

This brief focuses on healthcare workers in hospitals. Similar challenges exist in other 
regarding the mental health of communities and populations disproportionately impacted by COVID-19, including Black, Indigenous, and other racialized populations. The Working Group reports its findings to the public and the Science Table. Its findings are also summarized in Science Briefs.

Correspondence to: Secretariat of the Ontario COVID-19 Science Advisory Table (info@covid19-sciencetable.ca)

Copyright: 2021 Ontario COVID-19 Science Advisory Table. This is an open access document distributed under the terms of the Creative Commons Attribution License, which permits unrestricted use, distribution, and reproduction in any medium, provided that the original work is properly cited.

The views and findings expressed in this Science Brief are those of the authors and do not necessarily reflect the views of all of the members of the Ontario COVID-19 Science Advisory Table, its Working Groups, and its partners. healthcare settings (e.g., long-term care, primary health care, public health), which are not reviewed here.

\section{Questions}

What is burnout and what are the risk factors for burnout?

How has the prevalence of burnout changed during the COVID-19 pandemic for hospital-based healthcare workers?

Which hospital-based healthcare workers are at particular risk?

What interventions for burnout are supported by evidence?

What modifiable mediators of burnout are appropriate targets for intervention?

\section{Findings}

In spring 2020, the prevalence of severe burnout was 30\%-40\%. By spring 2021, rates $>60 \%$ were found in Canadian physicians, nurses, and other healthcare professionals.

Healthcare professionals in intensive care settings, COVID-19 units or hospitals, and emergency departments have had elevated risk of burnout compared to other hospital healthcare workers. Nurses and younger healthcare professionals or trainees have been at greater risk of burnout.

Organizational interventions have larger effects on reducing burnout than individual ones. Individual-level interventions include education and stress reduction techniques and should be complementary to organization-level interventions. Interventions that reduce burnout by even a small amount reduce adverse consequences.

Groups that have been most affected should be prioritized: nurses, those in intensive care and emergency departments, recent graduates and trainees.

Targets for intervention include (i) maintaining adequate staffing, (ii) leadership, (iii) work conditions, (iv) confidence in performing relevant tasks, (v) support networks, and (vi) moral distress (about constraints on doing the right thing).

System- and organization-level interventions to reduce burnout include visible and authentic senior leadership and managerial support, training to increase worker confidence with unfamiliar tasks, addressing workplace characteristics (e.g., overtime and scheduling shifts $>12$ hours) and supporting workers experiencing moral distress.

\section{Interpretation}

Hospital-based healthcare workers have experienced substantially increased burnout during the COVID-19 pandemic. Sustained burnout will likely contribute to staff retention challenges due to healthcare providers leaving their workplaces and professions. A vicious circle may be underway where understaffing leads to increased burnout and an even weaker healthcare workforce.

Maintaining the healthcare workforce will benefit from increasing the number of new graduates and by retaining current staff through financial compensation and fostering supportive workplace characteristics including supportive leadership at executive, director, and manager levels, continued professional development, effective communication, appropriate autonomy, and collegial relationships among workers and managers.

Optimal reduction and prevention of burnout depends on stronger evidence. Research evaluating organization and system-level interventions should be promoted.

\section{Background}

Burnout affects a wide range of healthcare workers, including those in hospitals, ${ }^{1,2}$ 
primary care and community settings, ${ }^{3-5}$ public health, ${ }^{6}$ long-term care, ${ }^{7}$ and first responders in emergency medical services. ${ }^{8,9}$ Each of these groups have been adversely affected by the COVID-19 pandemic. Given the diversity of these settings and of healthcare providers, this brief focuses on hospitals, where the evidence needed to assess the impact and to guide interventions is richer. However, many of the principles and recommendations may be applicable to those in practice in non-hospital settings.

\section{Questions}

What is burnout and what are the risk factors for burnout?

How has the prevalence of burnout changed during the COVID-19 pandemic for hospital-based healthcare workers?

Which hospital-based healthcare workers are at particular risk?

What interventions for burnout are supported by evidence?

What modifiable mediators of burnout are appropriate targets for intervention?

\section{Findings}

\section{Defining and Measuring Burnout}

Burnout is an occupational hazard caused by cumulative workplace stress that was recognized as a substantial problem in healthcare prior to the COVID-19 pandemic. It consists of emotional exhaustion (feeling drained and fatigued), depersonalization (becoming indifferent or emotionally distant), and a diminished sense of professional achievement. ${ }^{10}$

Burnout is associated with worse patient outcomes and reduced workplace satisfaction and productivity for healthcare professionals and trainees of all disciplines. For the healthcare system, burnout poses a risk to adequate staffing by contributing to absenteeism, higher workforce turnover, and greater likelihood that professionals will consider leaving their work. ${ }^{11-14}$ Burnout also results in lost productivity and is consistently correlated with depressive symptoms, ${ }^{15-18}$ and, in some cases, with thoughts of suicide. ${ }^{19}$ Emotional exhaustion, in particular, has been found to be associated with substance use and poor self-reported physical health in physicians, ${ }^{20}$ and with anxiety, lower self-esteem, and poor quality of life in nurses. ${ }^{17}$ Burnout of healthcare professionals and trainees is also associated with diminished safety, increased medical errors, reduced quality of care, and lower patient satisfaction. ${ }^{11,21-25}$

Rates of burnout have been measured as the rate of cases (prevalence) in crosssectional surveys of healthcare professionals in many settings. Although most studies survey nurses and/or physicians, studies of other healthcare professions have comparable findings. The prevalence of burnout is sensitive to how it is measured (e.g., scales and cut-off values used to define a case), ${ }^{26}$ which makes comparing the results of prevalence studies that have used different methodology challenging. This brief has been based on evidence that identified burnout as defined by the Maslach Burnout Inventory (MBI-EE), so as to identify data from the widest range of research using clear and replicable methods. The MBI-EE is a psychological assessment tool that measures an individual's experience of burnout along three dimensions: emotional exhaustion, depersonalization, and personal accomplishment. When reporting case rates we focus on burnout defined as a score $>26$ on the emotional exhaustion scale of the MBI-EE.

Prior to the COVID-19 pandemic, the prevalence of burnout in healthcare workers ranged from $<20 \%$ to $>50 \%,{ }^{27-29}$ with many studies of hospital-based nurses and physicians reporting rates of severe burnout in the range of $20-40 \%,{ }^{26}$ including meta-analyses of nurses in oncology (17 studies of 9959 RNs with severe burnout at 
$30 \%),{ }^{30}$ emergency medicine (13 studies of 1,566 RNs with severe burnout at $31 \%$ ), ${ }^{31}$ and primary care ( 8 studies of 1,110 RNs with severe burnout at $28 \%$ ) and physicians in emergency medicine (17 studies of $1,943 \mathrm{MDs}$ with severe burnout at $40 \%$ ). ${ }^{32,33}$ Collectively, the range of $20-40 \%$ serves as a benchmark for burnout identified using this case definition.

In a global meta-analysis of nurses (113 studies of 45,539 RNs), the prevalence of burnout in North American nurses was similar to that found in Europe, Latin American, and Central Asia, with the highest rates found in Southeast Asia and the lowest in Africa and the Middle East. Among specialties, the highest rates were in intensive/ critical care, emergency medicine, and pediatric nursing. ${ }^{34}$

\section{Risk Factors for Burnout}

Workplace-associated risk factors are related to the culture, environment and structure present in hospital workplaces. Figure 1 highlights the interplay between these factors, individual characteristics and burnout.

\section{CONTRIBUTING FACTORS}

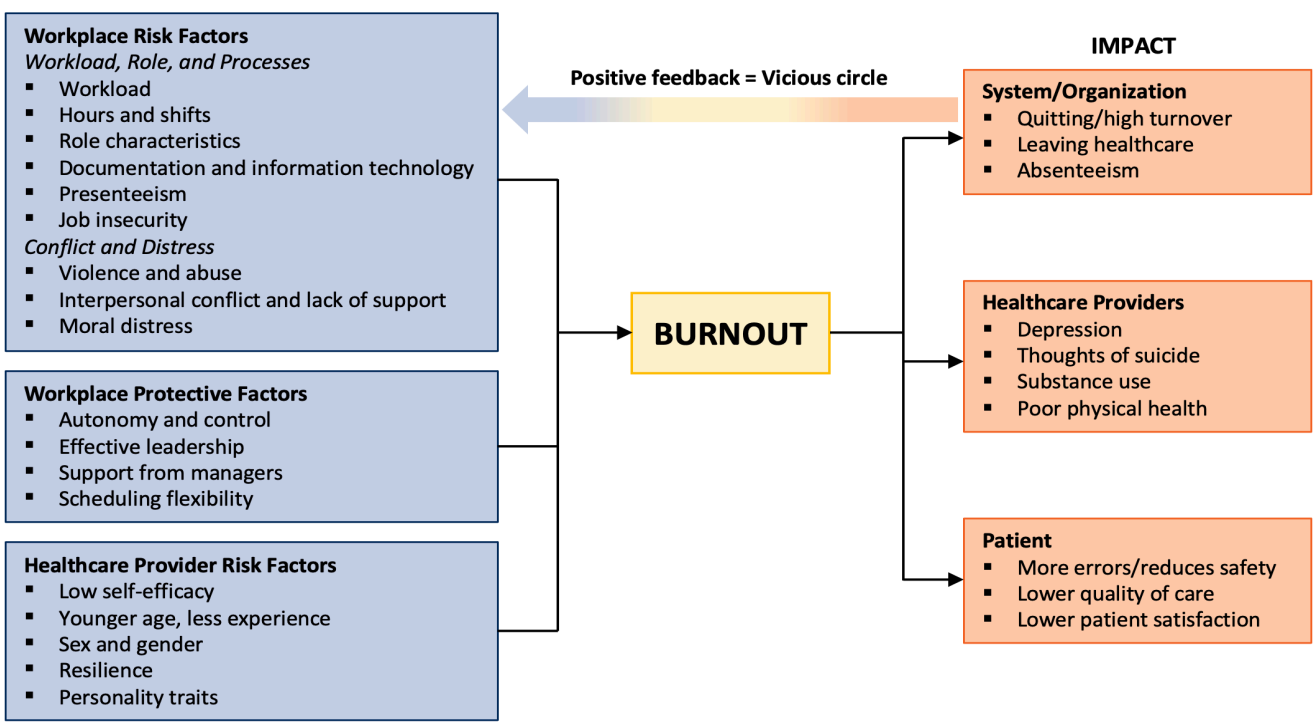

Figure 1. Burnout Framework

Review of the pre-COVID literature identified contributing factors, which are synthesized in this framework. These include workforce risk factors (e.g. workload, role and processes); workforce protective factors (e.g. autonomy and control, leadership, and scheduling flexibility); and healthcare provider (e.g. self-efficacy, age, gender, resilience, personality traits) emerged. This framework also includes the impact of burnout on organizations, healthcare providers, and patients.

\section{Workload}

Burnout is consistently associated with high workload. Approaches to operationalizing workload include measuring direct care time, clinician-patient ratio, patient acuity, and patient turnover. ${ }^{35}$ There is a strong relationship between nurse-patient ratio and burnout, ${ }^{1,11,36}$ but this ratio, in itself, does not consider patient acuity or the presence of supportive services, both of which contribute to workload. ${ }^{37} \mathrm{~A}$ cross-sectional study of 472 British Columbia nurses considered several aspects of workload and found patient acuity was associated with higher emotional exhaustion, whereas job satisfaction was associated with perceived heavy workload which was measured by asking about the frequency of factors such as arriving early/staying late and working through breaks. ${ }^{38}$ A similar finding was identified in a U.S. study. ${ }^{39}$ Studies of physicians also found a consistent relationship between burnout and workload, variously defined according to daily or weekly work hours, overnight duty, schedule inflexibility, patient load, or consultations per day or week. ${ }^{21}$

Importantly, to the extent that burnout challenges recruitment and retention of 
healthcare professionals, a vicious circle may arise in which understaffing is both a cause and consequence of burnout.

Hours and Shifts

As a short-term solution to nursing shortages, nurses are asked to work overtime on days off or to work extended hours. Working extended hours is linked to poor intershift recovery and fatigue, amplified stress responses, and increased likelihood of making errors, resulting in adverse patient outcomes. ${ }^{40-42}$ It has been recommended, based on a systematic review of nurse work schedules and adverse outcomes, that nursing shifts should not be longer than 12 hours. ${ }^{43}$ However, for medical residents, reducing shift length alone has not resulted in improved well-being. ${ }^{44}$

\section{Presenteeism}

Presenteeism is working when in poor mental or physical health. A prospective study of 258 hospital nurses over three years found that job demands, including workload and perceived demands from patients, contributed to working when sick, which in turn was associated with burnout. ${ }^{45}$

\section{Job Insecurity}

At times of restructuring and economic downturn, perceived job insecurity is associated with higher burnout. ${ }^{46}$ This may also be present for workers hired during the pandemic on a temporary basis.

\section{Role Characteristics}

Role conflict, significance, variety, and reward. Greater risk of burnout in nurses is associated with role conflict whereby workers have multiple roles which have contradictory, competing, or incompatible expectations and when there is ambiguity about role expectations. ${ }^{11}$ Roles characterized by low perceived task significance and variety, ${ }^{47-49}$ and high-effort/low reward are also associated with greater burnout. ${ }^{50-52}$

\section{Moral Distress}

Moral distress is the psychological state that arises when external constraints prevent one from pursuing the right course of action (e.g. when a shortage of resources prevents one from maintaining the usual standard of care or frequent contact with futile care in an ICU setting)..$^{53}$ In nurses, burnout is correlated with the frequency of such events and with experiencing moral distress. ${ }^{54-56}$

\section{Interpersonal Conflict and Lack of Support}

There is an association between interpersonal conflict and increased burnout. ${ }^{11,57-61}$ This conflict is more frequently documented occurring between colleagues with similar levels of authority, $57,58,62$ and in relationships with a power or hierarchy imbalance, such as nurse-physician or nurse-manager relationships. ${ }^{57-59}$ In addition, nurses and physicians who do not feel supported by their colleagues and managers have increased levels of burnout. ${ }^{11,21,58}$

\section{Violence and Abuse}

Various forms of violence and abuse in healthcare workplaces are common. A 2019 survey of 4462 British Columbia nurses reported these rates: emotional abuse $83 \%$, threat of assault $78 \%$, physical assault $67 \%$, verbal sexual harassment $55 \%$ and sexual assault $11 \% .{ }^{63}$ Similar rates were found in the U.S for nurses. ${ }^{64}$ Bullying is commonly experienced by young physicians, especially women (rates 30-95\% in a review of 18 studies). ${ }^{65}$ These experiences were consistently correlated with burnout. ${ }^{66-68}$ 
Documentation and Information Technology

Among physicians, burnout is more common among those who report having insufficient time for documentation (2.8 times odds ratio), spending excessive time on electronic health records at home (1.9 times odds ratio) or experiencing daily frustration with electronic health records (2.4 times odds ratio). ${ }^{69}$ In a survey of 5,197 U.S. physicians, physician-rated usability of electronic health records had a strong relationship with burnout. ${ }^{70}$

There is evidence of protective factors including culture, leadership and supportive systems alongside staff autonomy and control that can protect workers from burnout.

\section{Authentic Senior or Executive Leadership}

A meta-analysis and a systematic review of leadership style in nursing concluded that effective leadership is associated with lower burnout and other positive effects on nurses' well-being. ${ }^{71,72}$ Fourteen studies found protective effects of organizational leaders who are "authentic" (e.g., self-aware, transparent, ethical, respectful, and who seek information and insights from a variety of sources when making decisions). ${ }^{11,69,73}$ Authentic executive leadership was of increasing importance to a healthy workforce as healthcare complexity increased..$^{74}$

\section{Support from Managers}

Evidence for a relationship between feeling support from managers and lower burnout was provided by most studies of nurses, ${ }^{11}$ but not all, ${ }^{75}$ and was also been found for other healthcare professionals. ${ }^{76}$ Other qualities of nurse managers associated with lower burnout in the staff reporting to them were trustworthiness and perceived competence. ${ }^{11}$

\section{Autonomy and Control}

Studies of physicians, ${ }^{77}$ social workers, ${ }^{78}$ and nurses, ${ }^{11,79}$ have all found that a high level of autonomy or control over one's practice (e.g., the perception that nurses have the freedom to make important patient care and work decisions) was associated with less burnout, both in Ontario and in international studies. ${ }^{80-82}$

\section{Scheduling Flexibility}

Flexibility in scheduling, including the ability to schedule days off, was associated with lower burnout. ${ }^{11}$

There is limited evidence regarding characteristics of health care workers that predispose them to burnout. Fewer individual-level risk characteristics for burnout have been identified. Most are not readily modifiable (sex, gender, age, personality traits), although one modifiable characteristic has been identified, self-efficacy.

\section{Self-Efficacy}

This refers to specifically focused confidence: a person's beliefs about their capabilities to perform relevant tasks and to influence events. ${ }^{83}$ It can be measured as a general trait, or as self-efficacy with respect to a specific challenge (e.g., working in critical care nursing, doing healthcare work during a pandemic). Self-efficacy can be improved with training in specific, relevant tasks and challenges. For example, training in communication skills increases self-efficacy for that task, ${ }^{84}$ and training in managing interpersonal and coping challenges related to a pandemic increases pandemic selfefficacy. ${ }^{85}$ A 2016 meta-analysis of 57 studies involving 22,773 teachers, healthcare workers, and other professionals found an association of medium strength (effect size -0.33 ) between low self-efficacy and burnout. There was no difference between general and specific measures of self-efficacy. ${ }^{86}$ Studies specific to healthcare professionals replicate this result. ${ }^{18,87}$ 


\section{Gender}

Gender differences in burnout for healthcare professionals depend on context. For example, a meta-analysis shows that among nurses, men are at higher risk of burnout, ${ }^{30}$ whereas among physicians, women may be at higher risk. ${ }^{88} \mathrm{~A}$ meta-analysis found greater emotional exhaustion in women and greater depersonalization in men across disciplines, although both effects were small. ${ }^{89}$

\section{Age and Experience}

A systematic review of 41 studies of surgeons and a study of 473 general hospital nurses and nursing students each found that younger professionals and trainees were at elevated risk of burnout. This was consistent with the 2018 Canadian Medical Association survey, which found that residents had $48 \%$ higher risk of burnout than practicing physicians. ${ }^{90-92}$ Possible explanations which have been suggested included longer work hours, less autonomy and discretion, or a "survival" bias introduced because those with high burnout leave the profession. ${ }^{90}$

\section{Resilience}

This is the ability to "bounce back" after stress. It can be understood at organizational and individual levels. ${ }^{93}$ For individual healthcare workers, resilience has been conceptualized as a composite of attitudes (e.g., living authentically, maintaining perspective) and behavioural skills (e.g., managing stress, building social networks), and was associated with measures of health. ${ }^{94} \mathrm{~A}$ resilient organization is one that has matched job demands to resources for workers and fosters a culture of connection and transparency. Resilient organizations are well positioned to achieve strategic objectives and face challenges during crises. ${ }^{95}$

\section{Personality Traits}

There is some literature from psychology which suggests an association between burnout and personality traits. Personality traits including extraversion (level of energy and sociability), agreeableness (interpersonal skills to approach or reject others), and conscientiousness (energy and sociability) serve as protective factors. ${ }^{96,97}$

\section{COVID-19 has Exacerbated Risk Factors for Burnout and Increased Prevalence}

Many risk factors for burnout have been exacerbated during the pandemic, including increased patient acuity, understaffing due to staff with COVID-19 compatible symptoms, exposure leading to quarantine, increased overtime, presenteeism, reassignment to unfamiliar roles, and circumstances that provoke moral distress.

In addition, working in healthcare during the pandemic has introduced circumstances that are expected to contribute to burnout. These include health risk to oneself and one's family and uncertainty about infectious risks and precautions. Further public health measures such as school and business closures also may impact health care workers and create strain between work and personal or family obligations.

As burnout results from cumulative occupational stress, ${ }^{98}$ its impact is expected to increase over time during the pandemic, and appears to be doing so. Early in the pandemic, representative studies of hospital workers in April/May 2020 in Italy and Belgium reported severe emotional exhaustion (MBI-EE >26) in $32-41 \%,{ }^{99-101}$ which is similar to the pre-pandemic benchmark. A weekly survey of 231 Canadian emergency physicians for 10 weeks from March to May, 2020 found emotional exhaustion and depersonalization did not change, consistent with the expectation that COVID-19related burnout would build slowly. ${ }^{102}$

One study of intensive care nurses and physicians in the Netherlands reported that burnout had risen in the first wave of the pandemic (from November 2019 to 
April 2020). Using a different case definition, so rates are not comparable to others reported here, they found rates had risen from $26 \%$ to $38 \%$ in nurses and $13 \%$ to $29 \%$ in physicians. ${ }^{103}$

As the pandemic persists, increased levels of burnout are being reported. A survey of 250 internal medicine specialist physicians in two Vancouver hospitals conducted from August to October 2020, found a prevalence of severe emotional exhaustion (MBI$\mathrm{EE}>26$ ) of $63 \% .{ }^{104} \mathrm{~A}$ longitudinal survey of a single cohort of 422 staff representing diverse roles (nurses, doctors, professionals of other disciplines, support services, researchers, learners etc.) in a downtown Toronto hospital repeated three times at three-month intervals from November 2020 to May 2021 found high and rising burnout. The prevalence of severe emotional exhaustion (MBI-EE $>26$ ) in nurses was 54\% (Fall 2020), 62\% (Winter 2021), and 63\% (Spring 2021), while the prevalence in all other healthcare professionals at the same time points was $43 \%, 56 \%$, and $62 \%$ respectively (Hunter J, personal communication). ${ }^{105}$

\section{Nurses and Workers in Intensive Care and Emergency Department Settings are at Higher Risk of Burnout}

Identifying groups at higher risk may serve to focus or sequence interventions. Evidence is consistent across diverse settings that healthcare professionals in intensive care settings have been at higher risk during the pandemic compared to other healthcare settings. ${ }^{106-110}$ Working in a COVID-19 unit or hospital or in an emergency department has also been associated with elevated burnout. ${ }^{108,111,112}$

During the pandemic, higher levels of burnout have consistently been reported in nurses than in other hospital-based healthcare professionals in Canada and internationally. ${ }^{103,105,113-116}$ Younger healthcare professionals and trainees have also been at elevated risk in all healthcare disciplines. ${ }^{107,112,114,117-119}$ Healthcare workers' ethnicity, culture, and race have not received wide study. One Canadian study of internal medicine specialists found that visible minority physicians were more likely to report lower personal accomplishment than others, but these groups did not differ in emotional exhaustion. ${ }^{104}$

Regarding sex differences, women healthcare personnel (including nurses, physicians, pharmacists, and other healthcare workers) have had higher emotional exhaustion, ${ }^{104,112,114,117,119-121}$ whereasmalesreporthigherdepersonalization. ${ }^{112,114,117,121,122}$ Regarding gender, a study of U.S. anesthetists in March 2020 found that those who identify as LGBTQ2S+ experienced greater burnout. ${ }^{123}$ Similarly, burnout was greater in individuals identifying as having non-binary gender in a nationwide U.S. survey of diverse healthcare professions between May and October 2020.124 Among internal medicine specialist physicians at two Vancouver hospitals, there was no difference in emotional exhaustion or depersonalization by sexual orientation. ${ }^{104}$

\section{Evidence-Based Interventions for Burnout}

Systematic reviews and meta-analyses summarize evidence for interventions at the level of organizations, individual interventions, and their combination. ${ }^{2,125,126}$ Since most known risk factors for burnout occur at the organization-level, it is consistent that a pre-pandemic meta-analysis of interventions for physicians found that organizational interventions were more effective than individual interventions (organizational mean difference $-12.46[-17.47,-7.4595 \% \mathrm{Cl}]$, individual mean difference $-3.36[-9.90,3.17$ $95 \% \mathrm{Cl}, \mathrm{p}=0.03) .{ }^{2}$ The interventions included shortened length of rotations for attending staff, reduced hours of duty shifts, and modifications to clinical work processes (e.g., communication improvements through monthly clinical meetings; medical assistants to enter electronic health record data; automating prescriptions). Another metaanalysis suggested the combination of organizational and individual interventions resulted in larger and/or longer-lasting benefits. ${ }^{127}$ The interventions reviewed in 
these meta-analyses are too heterogeneous to support specific recommendations. ${ }^{128}$

Professional organizations have also emphasized the importance of system level responses to burnout, including streamlining documentation, ensuring equitable compensation, and promoting the seamless integration of digital tools. ${ }^{3}$

Despite substantially less evidence for individual-level burnout risk factors, individual interventions are more widely studied. On the whole, studies of these interventions indicate that they provide significant, but only moderately large, short-term benefits, with no evidence to support greater effectiveness of any particular intervention.,125 Those studied include small group education, stress management/self-care (yoga, mindfulness, relaxation techniques), communication skills training, coping skills training. ${ }^{2,125}$ Regarding prevention, cognitive behavioural therapy interventions have resulted in modest benefits with small to medium effect sizes. ${ }^{125}$

Importantly, interventions that reduce burnout by even a small amount are meaningfully associated with reducing adverse consequences of burnout including healthcare system turnover and absenteeism; ;,11,129 medical errors, reduced patient safety and satisfaction; ${ }^{25}$ healthcare provider depression, ${ }^{17}$ suicide, ${ }^{130}$ substance use, and decreased physical health. ${ }^{20}$

\section{Modifiable Mediators of Burnout that are Appropriate Targets for Intervention}

Given the limitations of evidence for specific interventions, we have reviewed the literature to identify the most plausible modifiable risk factors for pandemic-related burnout, based on consistent evidence of association with burnout prior to COVID-19 and evidence of correlation with burnout during the pandemic.

\section{Work Conditions, Workload, and Fatigue}

Longer shifts (more than 12 hours, ${ }^{131}$ for nurses) and a greater workload were associated with increased burnout in physicians and nurses in several settings during the pandemic. ${ }^{99,112,118,132}$ A meta-analysis of studies of nurses spanning 11 countries among Europe, Asia, and North America during COVID-19 identified higher burnout in settings that reported insufficient material resources and understaffing. ${ }^{112}$ Poor sleep and fatigue have also been identified as potential exacerbating factors. ${ }^{105,116,121}$

\section{Self-Efficacy}

In a study of 2,014 nurses in hospitals in Wuhan, China in February, 2020, both general self-efficacy and specific confidence in one's own and one's institution's readiness to deal with COVID-19 were associated with lower burnout. ${ }^{106}$ A Romanian study found that lower self-efficacy, in addition to a lack of access to training, professional development, and continuing education, were significant predictors of burnout during the COVID-19 pandemic. ${ }^{133}$ In a Toronto hospital, self-efficacy with respect to pandemicrelated challenges (e.g. "How confident are you that you will be able to perform duties that are outside your usual job") in Fall 2020 was a strong predictor of lower burnout at two time points over the following six months in healthcare professionals of all disciplines. ${ }^{105}$ A meta-analysis of nurses found lower levels of specialized training were associated with greater burnout. ${ }^{112}$

\section{Moral Distress}

Moral distress has been a prominent theme for healthcare workers during the pandemic. ${ }^{134-137}$ In a study in the Netherlands, two sources of moral distress that increased in COVID-19 (scarcity of resources and the perception of colleagues acting unsafely) were significant predictors of burnout. ${ }^{103}$ In a Toronto hospital, moral distress was strongly related to emotional exhaustion and accounted for most of the difference in burnout between roles (nurses vs. other health professionals vs. other staff with regular patient contact; Hunter J, personal communication). Interventions that have 
been suggested to reduce the harmful effects of moral distress include education about the concept and critical reflective practice that allows cognitive reframing. ${ }^{138}$ More research is needed to evaluate such interventions.

\section{Support Networks}

Insufficient support at work, in the form of peer social support or institutional support, has been a risk factor for burnout during COVID-19. ${ }^{112,123}$ Support from leadership or supervisors, in the form of feedback and recognition, has also mediated burnout. ${ }^{133}$ In a study of Malaysian healthcare workers, increased burnout during COVID-19 was associated with perceived inadequate psychosocial support at work and feelings of stretched work relationships with superiors and colleagues. ${ }^{139}$

\section{Interpretation}

Burnout was a significant problem for hospital-based healthcare workers prior to the COVID-19 pandemic and has increased substantially. Multiple Canadian studies indicate that by the fall of 2020 and afterwards, more than $60 \%$ of healthcare workers surveyed were experiencing severe emotional exhaustion.

It is not known how long elevated levels of burnout will persist. Two considerations suggest that elevated levels of burnout will not resolve quickly: (1) After the severe acute respiratory syndrome (SARS) outbreak of 2003, which was much more circumscribed in scope and severity, elevated burnout and other indicators of chronic stress persisted in affected healthcare workers for as long as they were studied (18-24 months); ${ }^{140}(2)$ the pre-pandemic benchmark of $20-40 \%$ of hospital based healthcare workers with severe emotional exhaustion suggests that a "return to normal" would be a return to conditions that are not optimal for recovery.

A marked and sustained increase in burnout is likely to lead healthcare professionals to seek work that involves less direct patient contact, shorter or more predictable hours, or to leave the profession altogether. The likelihood of a workforce reduction is supported by survey data indicating that $43 \%$ of members of the 1,716 Registered Nurses Association of Ontario members surveyed in January-February 2021 were considering leaving nursing after the pandemic (27\% somewhat likely, $7 \%$ likely, $9 \%$ very likely). ${ }^{141}$ This concern is also supported by a survey of 131 physicians and nurses in a Montreal hospital where the intention to quit was reported by $50 \%$ of nurses and $20 \%$ of physicians and a survey of 257 physicians in Vancouver of whom 21\% were considering quitting. ${ }^{104,113}$ In a Toronto hospital in Spring 2021, 30\% of nurses and $13 \%$ of physicians surveyed reported considering leaving their jobs, specifically because of moral distress (Hunter J, personal communication). Observation indicates that professionals are acting on their intention to leave. Media accounts have reported many Canadian nurses leaving their profession as a result of COVID-19 stresses, ${ }^{142}$ emergency department closures due to understaffing, ${ }^{143-146}$ and an Ontario hospital emergency department offering a large "signing bonus" to attract new nurses. ${ }^{147}$

A vicious circle of understaffing leading to burnout, which in turn leads to further challenges to recruitment and retention creates the potential for a sustained and worsening problem in healthcare, which may be underway. A further challenge is that these conditions are present outside Ontario as well, indeed globally, which reduces the pool of healthcare workers available to replace those who reduce or cease direct patient care.

\section{Multi-Faceted Mitigation Strategies are Needed for Burnout}

The below recommendations align with a previous review of best practices. ${ }^{148} \mathrm{~A}$ number of professional organizations have developed recommendations for system level responses to burnout. While it is challenging to assess the evidence of impact 
of such recommendations, they are worth noting given the challenges identified in this document. In August, 2021 the Ontario Medical Association (OMA) Burnout Task Force released 5 system level recommendations to physician burnout which highlighted the need to reduce documentation and administrative work, ${ }^{3}$ fairly and equitably compensate work, make organizational policy changes to improve worklife balance, promote integration of digital health tools into workflows and provide institutional wellness supports.

The Registered Nurses' Association of Ontario (RNAO) released a series of recommendations related to nurse well-being and burnout related to increasing support for early, mid-career and senior nursing positions, increase overall staffing of nurses, bolster admission to nursing programs to ensure adequate workforce and increase nurse practitioner positions. ${ }^{141}$

In the long-term, maintaining an adequate healthcare workforce will benefit from support for an expansion of training opportunities to increase the number of new graduates. Planning for adequate health human resources will necessitate interministerial collaboration (including, for example, the Ministry of Colleges and Universities) to ensure that university and college-based training programs tasked with producing the next generations of healthcare workers (e.g., nurses, physicians, psychologists, and other allied healthcare workers) are able to meet the needs of the broader health system and patient needs. The health human resources strategy can also recognize risks to a healthy workforce such as burnout, ${ }^{149}$ and mitigate healthcare worker shortages due to changing careers, and the impending health human resource crisis as a consequence of retirements due to an aging workforce.

Given evidence that high stress and the intention to leave nursing is particularly common among recent graduates, ${ }^{150-152}$ it is relevant that transition to practice programs consisting of formal teaching, preceptorship, and mentorship, lasting 27-52 weeks, appear to promote retention and reducing turnover among novice nurses. ${ }^{153}$

In an environment in which there is competition between organizations for staff, financial compensation is likely to influence where professionals choose to work.

Regarding opportunities to make the workplace more attractive, there is clear evidence for the "magnet" characteristics which lead nurses to choose one workplace over another. These include: nursing leadership which is visible, supportive, visionary, and holds high status in the organization; processes for continuing professional development; clear communication and responsiveness from leaders; a relatively flat organizational hierarchy, local autonomy, and control of decision-making and scheduling; and collegial nurse-physician relationships. ${ }^{154}$ Importantly, magnet characteristics are also associated with lower burnout and better patient outcomes..$^{155,156}$

\section{Individual-Level Interventions to Reduce Burnout}

Individual-level interventions to reduce burnout should be complemented by organizational interventions. There is no evidence for the superiority of any one type of intervention and so it is recommended to provide access to a variety of evidencesupported resources. Interventions that have been supported in well-designed research trials include small group education, stress management, yoga, mindfulness, relaxation techniques, communication skills training, coping skills training, and cognitive-behavioural therapy interventions.

Providing individual resources to professionals who have been harmed by circumstances beyond their control risks the perception of assigning responsibility for repair to the person who has been harmed (e.g., blaming the victim). Individual-level interventions should be understood to be complementary to organization and system level approaches. 
Further research should be promoted to determine if understanding individuals' context (e.g. family circumstances, race, ethnicity) would allow for effectively tailored interventions.

\section{Organizational-Level Interventions}

The most appropriate targets for system- and organization-level interventions are addressing shift length and scheduling, ensuring adequate training, offering education and support for moral distress.

\section{Shift Length and Scheduling}

Shifts should not be longer than 12 hours for nurses, and overtime hours should be avoided. Scheduling should ensure adequate inter-shift recovery. Avoiding overtime hours requires maintaining sufficient numbers of staff. For all disciplines, appropriate levels of staffing depend on numerous factors including patient acuity, direct care time, and availability of supportive services. Ongoing monitoring and evaluation of workload is necessary to inform shift length, scheduling, and staffing levels.

\section{Training and Self-Efficacy}

Training and support should be provided to promote healthcare providers' competence and confidence with respect to (1) unfamiliar work tasks, such as those that result from reassignment to new areas, and (2) challenges that are directly related to burnout, such as managing interpersonal conflict, dealing with workplace violence and abuse, and responding to moral distress.

\section{Leadership}

Leaders have a critical role in creating a supportive work environment and culture. ${ }^{157}$ Leaders should be visible, transparent, ethical, respectful, and should seek information from a variety of sources, including frontline staff, when making decisions. Support of staff by managers is crucial and should be promoted by organizational leaders. Such support may include fostering a culture that promotes well-being (e.g. discourages presenteeism), actively working to reduce systemic contributors to burnout, openness and responsiveness to feedback, and recognition of achievements and sacrifices.

\section{Support Networks}

A positive workplace culture should facilitate mutual support between colleagues. The development of peer support programs is also recommended. In addition, individual psychological assessment and treatment should be available for those who wish it.

\section{Moral Distress}

Organizations should recognize that the COVID-19 pandemic has increased healthcare professionals' exposure to moral distress. Although interventions to reduce the harmful effects of moral distress are not yet well-studied, education about the concept and group-based critical reflective practice that allows cognitive reframing may be valuable.

Interventions to reduce burnout should be first directed towards groups who have been most affected. These include nurses, early career professionals and trainees, healthcare workers in intensive care and emergency departments, and those who have worked in high acuity settings with large numbers of COVID-19 patients or outbreaks.

\section{Methods Used for This Science Brief}

We searched PubMed, Google Scholar, the COVID-19 Rapid Evidence Reviews, LitCovid in PubMed, and the World Health Organization's Global Literature on Coronavirus Disease. In addition, we retrieved reports citing relevant articles through Google Scholar 
and reviewed references from identified articles for additional studies. Selected media reports of recent trends were included when relevant. The search was last updated on [July 31, 2021]. The COVID-19 Evidence Synthesis Network performed a research evidence scan for this Science Brief, published in an Evidence Synthesis Briefing Note. The COVID-19 Evidence Synthesis Network is comprised of organizations in Ontario's evidence synthesis and knowledge translation community who collectively provide high-quality, relevant, and timely synthesized research evidence about COVID-19.

\section{Author Contributions}

The Mental Health Working Group conceived the Science Brief. RGM, NDH, GS, HDS wrote the first draft of the Science Brief. LJ, CM and LM reviewed and revised the draft. All authors revised the Science Brief critically for important intellectual content and approved the final version.

\section{References}

1. Aiken LH, Clarke SP, Sloane DM, Sochalski J, Silber JH. Hospital nurse staffing and patient mortality, nurse burnout, and job dissatisfaction. JAMA. 2002;288(16):19871993. https://doi.org/10.1001/jama.288.16.1987

2. West $C P$, Dyrbye LN, Erwin PJ, Shanafelt TD. Interventions to prevent and reduce physician burnout: A systematic review and meta-analysis. The Lancet. 2016;388(10057):2272-2281. https://doi.org/10.1016/S0140-6736(16)31279-X

3. Ontario Medical Association. Healing the Healers: System-Level Solutions to Physician Burnout. Ontario Medical Association; 2021:48. https://www.oma.org/ uploadedfiles/oma/media/pagetree/advocacy/health-policy-recommendations/ burnout-paper.pdf

4. Quick COVID-19 survey. The Larry A. Green Center. Published 2021. https://www. green-center.org/covid-survey

5. Vassos M, Nankervis K, Skerry T, Lante K. Can the job demand-control-(support) model predict disability support worker burnout and work engagement? J Intellect Dev Disabil. 2019;44(2):139-149. https://doi.org/10.3109/13668250.2017.13108 18

6. Bryant-Genevier J. Symptoms of depression, anxiety, post-traumatic stress disorder, and suicidal ideation among state, tribal, local, and territorial public health workers during the COVID-19 pandemic - United States, March-April 2021. MMWR Morb Mortal Wkly Rep. 2021;70. https://doi.org/10.15585/mmwr. $\mathrm{mm} 7026 \mathrm{e} 1$

7. Tanaka K, Iso N, Sagari A, et al. Burnout of long-term care facility employees: Relationship with employees' expressed emotion toward patients. Int J Gerontol. 2015;9(3):161-165. https://doi.org/10.1016/j.ijge.2015.04.001

8. Boland LL, Kinzy TG, Myers RN, et al. Burnout and exposure to critical incidents in a cohort of emergency medical services workers from Minnesota. West J Emerg Med Integrating Emerg Care Popul Health. 2018;19(6). https://doi.org/10.5811/ westjem.8.39034

9. Koval KW, Lindquist B, Gennosa C, et al. First look at emergency medical technician wellness in India: Application of the Maslach burnout Inventory in an unstudied population. PloS One. 2020;15(3). https://doi.org/10.1371/journal.pone.0229954

10. Maslach C, Jackson SE, Leiter MP. Maslach burnout inventory manual. Published 1996. https://www.worldcat.org/title/maslach-burnout-inventory-manual/ oclc/807246527 
11. Dall'Ora C, Ball J, Reinius $M$, Griffiths $P$. Burnout in nursing: $A$ theoretical review. Hum Resour Health. 2020;18(1):41. https://doi.org/10.1186/s12960-020-004699

12. Grossman Z, Chodick G, Kushnir T, Cohen HA, Chapnick G, Ashkenazi S. Burnout and intentions to quit the practice among community pediatricians: Associations with specific professional activities. Isr J Health Policy Res. 2019;8(1):2. https:// doi.org/10.1186/s13584-018-0268-2

13. Heinen MM, van Achterberg T, Schwendimann R, et al. Nurses' intention to leave their profession: A cross sectional observational study in 10 European countries. Int J Nurs Stud. 2013;50(2):174-184. https://doi.org/10.1016/j.ijnurstu.2012.09.019

14. Willard-Grace R, Knox M, Huang B, Hammer H, Kivlahan C, Grumbach K. Burnout and health care workforce turnover. Ann Fam Med. 2019;17(1):36-41. https://doi. org/10.1370/afm.2338

15. Dewa CS, Loong D, Bonato S, Thanh NX, Jacobs P. How does burnout affect physician productivity? A systematic literature review. BMC Health Serv Res. 2014;14(1):325. https://doi.org/10.1186/1472-6963-14-325

16. Bianchi R, Boffy C, Hingray C, Truchot D, Laurent E. Comparative symptomatology of burnout and depression. J Health Psychol. 2013;18(6):782-787. https://doi. org/10.1177/1359105313481079

17. Choi B-S, Kim JS, Lee D-W, et al. Factors associated with emotional exhaustion in South Korean nurses: A cross-sectional study. Psychiatry Investig. 2018;15(7):670676. https://doi.org/10.30773/pi.2017.12.31

18. Smeds MR, Janko MR, Allen S, et al. Burnout and its relationship with perceived stress, self-efficacy, depression, social support, and programmatic factors in general surgery residents. Am J Surg. 2020;219(6):907-912. https://doi.org/10.1016/j. amjsurg.2019.07.004

19. Dyrbye LN, Thomas MR, Massie FS, et al. Burnout and suicidal ideation among U.S. medical students. Ann Intern Med. 2008;149(5):334-341. https://doi. org/10.7326/0003-4819-149-5-200809020-00008

20. Williams ES, Rathert C, Buttigieg SC. The personal and professional consequences of physician burnout: A systematic review of the literature. Med Care Res Rev. 2020;77(5):371-386. https://doi.org/10.1177/1077558719856787

21. Azam K, Khan A, Alam MT. Causes and adverse impact of physician burnout: A systematic review. J Coll Physicians Surg--Pak JCPSP. 2017;27(8):495-501. https:// www.jcpsp.pk/archive/2017/Aug2017/10.pdf

22. Hall LH, Johnson J, Watt I, Tsipa A, O'Connor DB. Healthcare staff wellbeing, burnout, and patient safety: A systematic review. PloS One. 2016;11(7):e0159015. https://doi.org/10.1371/journal.pone.0159015

23. Panagioti M, Geraghty K, Johnson J, et al. Association between physician burnout and patient safety, professionalism, and patient satisfaction: A systematic review and meta-analysis. JAMA Intern Med. 2018;178(10):1317-1331. https://doi. org/10.1001/jamainternmed.2018.3713

24. Poghosyan L, Clarke SP, Finlayson M, Aiken LH. Nurse burnout and quality of care: Cross-national investigation in six countries. Res Nurs Health. 2010;33(4):288-298. https://doi.org/10.1002/nur.20383

25. Shanafelt TD, Balch CM, Bechamps G, et al. Burnout and medical errors among American surgeons. Ann Surg. 2010;251(6):995-1000. https://doi.org/10.1097/ SLA.0b013e3181bfdab3 
26. Rotenstein LS, Torre M, Ramos MA, et al. Prevalence of burnout among physicians: A systematic review. JAMA. 2018;320(11):1131-1150. https://doi.org/10.1001/ jama.2018.12777

27. Goehring C, Bouvier Gallacchi M, Künzi B, Bovier P. Psychosocial and professional characteristics of burnout in Swiss primary care practitioners: A cross-sectional survey. Swiss Med Wkly. 2005;135(7-8):101-108. https://doi.org/2005/07/smw10841

28. Pedersen AF, Andersen CM, Olesen F, Vedsted P. Risk of burnout in Danish GPs and exploration of factors associated with development of burnout: A two-wave panel study. Int J Fam Med. 2013;2013:e603713. https://doi.org/10.1155/2013/603713

29. Busis NA, Shanafelt TD, Keran CM, et al. Burnout, career satisfaction, and wellbeing among US neurologists in 2016. Neurology. 2017;88(8):797-808. https:// doi.org/10.1212/WNL.0000000000003640

30. Cañadas-De la Fuente GA, Gómez-Urquiza JL, Ortega-Campos EM, Cañadas GR, Albendín-García L, De la Fuente-Solana El. Prevalence of burnout syndrome in oncology nursing: A meta-analytic study. Psychooncology. 2018;27(5):1426-1433. https://doi.org/10.1002/pon.4632

31. Gómez-Urquiza JL, De la Fuente-Solana El, Albendín-García L, Vargas-Pecino C, Ortega-Campos EM, Cañadas-De la Fuente GA. Prevalence of burnout syndrome in emergency nurses: A meta-analysis. Crit Care Nurse. 2017;37(5):e1-e9. https:// doi.org/10.4037/ccn2017508

32. Monsalve-Reyes CS, San Luis-Costas C, Gómez-Urquiza JL, Albendín-García L, Aguayo R, Cañadas-De la Fuente GA. Burnout syndrome and its prevalence in primary care nursing: A systematic review and meta-analysis. BMC Fam Pract. 2018;19(1):59. https://doi.org/10.1186/s12875-018-0748-z

33. Zhang, Qin, Mu, Ming-chun, He, Yan, Cai, Zhao-lun, Li, Zheng-chi. Burnout in emergency medicine physicians: A meta-analysis and systematic review. Medicine (Baltimore). 2020;99(32):e21462. https://doi.org/10.1097/ MD.0000000000021462

34. Woo T, Ho R, Tang A, Tam W. Global prevalence of burnout symptoms among nurses: A systematic review and meta-analysis. J Psychiatr Res. 2020;123:9-20. https://doi.org/10.1016/j.jpsychires.2019.12.015

35. Fishbein D, Nambiar S, McKenzie K, et al. Objective measures of workload in healthcare: A narrative review. Int J Health Care Qual Assur. 2019;33(1):1-17. https://doi.org/10.1108/IJHCQA-12-2018-0288

36. Pérez-Francisco DH, Duarte-Clíments G, del Rosario-Melián JM, Gómez-Salgado J, Romero-Martín M, Sánchez-Gómez MB. Influence of workload on primary care nurses' health and burnout, patients' safety, and quality of care: Integrative review. Healthcare. 2020;8(1):12. https://doi.org/10.3390/healthcare8010012

37. Wynendaele $\mathrm{H}$, Willems R, Trybou J. Systematic review: Association between the patient-nurse ratio and nurse outcomes in acute care hospitals. J Nurs Manag. 2019;27(5):896-917. https://doi.org/10.1111/jonm.12764

38. MacPhee M, Dahinten VS, Havaei F. The impact of heavy perceived nurse workloads on patient and nurse outcomes. Adm Sci. 2017;7(1):7. https://doi.org/10.3390/ admsci7010007

39. Phillips C. Relationships between workload perception, burnout, and intent to leave among medical-surgical nurses. JBI Evid Implement. 2020;18(2):265-273. https://doi.org/10.1097/XEB.0000000000000220 
40. Bernstrøm $\mathrm{VH}$, Alves $\mathrm{DE}$, Ellingsen $\mathrm{D}$, Ingelsrud $\mathrm{MH}$. Healthy working time arrangements for healthcare personnel and patients: A systematic literature review. BMC Health Serv Res. 2019;19(1):193. https://doi.org/10.1186/s12913019-3993-5

41. Dall'Ora C, Griffiths P, Ball J, Simon M, Aiken LH. Association of $12 \mathrm{~h}$ shifts and nurses' job satisfaction, burnout and intention to leave: Findings from a crosssectional study of 12 European countries. BMJ Open. 2015;5(9):e008331. https:// doi.org/10.1136/bmjopen-2015-008331

42. Sagherian K, Clinton ME, Abu-Saad Huijer H, Geiger-Brown J. Fatigue, work schedules, and perceived performance in bedside care nurses. Workplace Health Saf. 2017;65(7):304-312. https://doi.org/10.1177/2165079916665398

43. Bae S-H. Relationships between comprehensive characteristics of nurse work schedules and adverse patient outcomes: A systematic literature review. J Clin Nurs. 2021;30(15-16):2202-2221. https://doi.org/10.1111/jocn.15728

44. Bolster $L$, Rourke $L$. The effect of restricting residents' duty hours on patient safety, resident well-being, and resident education: An updated systematic review. J Grad Med Educ. 2015;7(3):349-363. https://doi.org/10.4300/JGME-D-14-00612.1

45. Demerouti E, Le Blanc PM, Bakker AB, Schaufeli WB, Hox J. Present but sick: A three-wave study on job demands, presenteeism and burnout. Career Dev Int. 2009;14(1):50-68. https://doi.org/10.1108/13620430910933574

46. Burke RJ, Ng ESW, Wolpin J. Economic austerity and healthcare restructuring: Correlates and consequences of nursing job insecurity. Int J Hum Resour Manag. 2015;26(5):640-656. https://doi.org/10.1080/09585192.2014.921634

47. Jansen PGM, Kerkstra A, Abu-Saad HH, Van Der Zee J. The effects of job characteristics and individual characteristics on job satisfaction and burnout in community nursing. Int J Nurs Stud. 1996;33(4):407-421. https://doi. org/10.1016/0020-7489(95)00060-7

48. Roelen CAM, Koopmans PC, Groothoff JW. Which work factors determine job satisfaction? Work Read Mass. 2008;30(4):433-439. https://pubmed.ncbi.nlm. nih.gov/18725706/

49. Zacher H, Dirkers BT, Korek S, Hughes B. Age-differential effects of job characteristics on job attraction: A policy-capturing study. Front Psychol. 2017;8:1124. https:// doi.org/10.3389/fpsyg.2017.01124

50. Basińska BA, Wilczek-Rużyczka E. The role of rewards and demands in burnout among surgical nurses. Int J Occup Med Environ Health. 2013;26(4):593-604. https://doi.org/10.2478/s13382-013-0129-8

51. Colindres C v., Bryce E, Coral-Rosero P, Ramos-Soto R m., Bonilla F, Yassi A. Effect of effort-reward imbalance and burnout on infection control among Ecuadorian nurses. Int Nurs Rev. 2018;65(2):190-199. https://doi.org/10.1111/inr.12409

52. He Y, Pang $Y$, Zhang $Y$, Fielding $R$, Tang L. Dual role as a protective factor for burnoutrelated depersonalization in oncologists. Psychooncology. 2017;26(8):1080-1086. https://doi.org/10.1002/pon.4425

53. Jameton A. Nursing practice: The ethical issues. Published 1984. https://www. worldcat.org/title/nursing-practice-the-ethical-issues/oclc/9896319

54. Fumis RRL, Junqueira Amarante GA, de Fátima Nascimento A, Vieira Junior JM. Moral distress and its contribution to the development of burnout syndrome among critical care providers. Ann Intensive Care. 2017;7(1):71. https://doi. org/10.1186/s13613-017-0293-2 
55. Lamiani G, Borghi L, Argentero P. When healthcare professionals cannot do the right thing: A systematic review of moral distress and its correlates. J Health Psychol. 2017;22(1):51-67. https://doi.org/10.1177/1359105315595120

56. Meltzer LS, Huckabay LM. Critical care nurses' perceptions of futile care and its effect on burnout. Am J Crit Care. 2004;13(3):202-208. https://doi.org/10.4037/ ajcc2004.13.3.202

57. Blackstock S, Salami B, Cummings GG. Organisational antecedents, policy and horizontal violence among nurses: An integrative review. J Nurs Manag. 2018;26(8):972-991. https://doi.org/10.1111/jonm.12623

58. Dubale BW, Friedman LE, Chemali Z, et al. Systematic review of burnout among healthcare providers in sub-Saharan Africa. BMC Public Health. 2019;19(1):1247. https://doi.org/10.1186/s12889-019-7566-7

59. Guidroz AM, Wang M, Perez LM. Developing a model of source-specific interpersonal conflict in health care. Stress Health. 2012;28(1):69-79. https://doi. org/10.1002/smi.1405

60. Spence Laschinger HK, Wong CA, Grau AL. The influence of authentic leadership on newly graduated nurses' experiences of workplace bullying, burnout and retention outcomes: A cross-sectional study. Int J Nurs Stud. 2012;49(10):12661276. https://doi.org/10.1016/j.ijnurstu.2012.05.012

61. Laschinger HKS, Read EA. The effect of authentic leadership, person-job fit, and civility norms on new graduate nurses' experiences of coworker incivility and burnout. JONA J Nurs Adm. 2016;46(11):574-580. https://doi.org/10.1097/ NNA.0000000000000407

62. Stanley KM, Martin MM, Michel Y, Welton JM, Nemeth LS. Examining lateral violence in the nursing workforce. Issues Ment Health Nurs. 2007;28(11):12471265. https://doi.org/10.1080/01612840701651470

63. Havaei F, MacPhee M, Ma A. Workplace violence among British Columbia nurses across different roles and contexts. Healthcare. 2020;8(2):98. https://doi. org/10.3390/healthcare8020098

64. Ulrich B, Barden C, Cassidy L, Varn-Davis N. Critical care nurse work environments 2018: Findings and implications. Crit Care Nurse. 2019;39(2):67-84. https://doi. org/10.4037/ccn2019605

65. Samsudin EZ, Isahak M, Rampal S. The prevalence, risk factors and outcomes of workplace bullying among junior doctors: A systematic review. Eur J Work Organ Psychol. 2018;27(6):700-718. https://doi.org/10.1080/1359432X.2018.1502171

66. Havaei F, Astivia OLO, MacPhee M. The impact of workplace violence on medical-surgical nurses' health outcome: A moderated mediation model of work environment conditions and burnout using secondary data. Int J Nurs Stud. 2020;109:103666. https://doi.org/10.1016/j.ijnurstu.2020.103666

67. Liu W, Zhao S, Shi L, et al. Workplace violence, job satisfaction, burnout, perceived organisational support and their effects on turnover intention among Chinese nurses in tertiary hospitals: A cross-sectional study. BMJ Open. 2018;8(6):e019525. https://doi.org/10.1136/bmjopen-2017-019525

68. Yoon HS, Sok SR. Experiences of violence, burnout and job satisfaction in Korean nurses in the emergency medical centre setting. Int J Nurs Pract. 2016;22(6):596604. https://doi.org/10.1111/ijn.12479

69. Gardner WL, Cogliser CC, Davis KM, Dickens MP. Authentic leadership: A review of the literature and research agenda. Leadersh Q. 2011;22(6):1120-1145. https:// 
doi.org/10.1016/j.leaqua.2011.09.007

70. Melnick ER, Dyrbye LN, Sinsky CA, et al. The association between perceived electronic health record usability and professional burnout among US physicians. Mayo Clin Proc. 2020;95(3):476-487. https://doi.org/10.1016/j. mayocp.2019.09.024

71. Wei $H$, King A, Jiang $Y$, Sewell KA, Lake DM. The impact of nurse leadership styles on nurse burnout: A systematic literature review. Nurse Lead. 2020;18(5):439450. https://doi.org/10.1016/j.mnl.2020.04.002

72. Alilyyani B, Wong CA, Cummings G. Antecedents, mediators, and outcomes of authentic leadership in healthcare: A systematic review. Int J Nurs Stud. 2018;83:34-64. https://doi.org/10.1016/j.ijnurstu.2018.04.001

73. Boamah SA, Laschinger $\mathrm{H}$. The influence of areas of worklife fit and work-life interference on burnout and turnover intentions among new graduate nurses. J Nurs Manag. 2016;24(2):E164-E174. https://doi.org/10.1111/jonm.12318

74. Jeffs L. Achieving the quadruple aim in healthcare: The essential role of authentic, complex and resilient nurse leaders. Nurs Leadersh. 2018;31(2). https://www. longwoods.com/content/25607/nursing-leadership/achieving-the-quadrupleaim-in-healthcare-the-essential-role-of-authentic-complex-and-resilient-n

75. Li B, Bruyneel L, Sermeus W, et al. Group-level impact of work environment dimensions on burnout experiences among nurses: A multivariate multilevel probit model. Int J Nurs Stud. 2013;50(2):281-291. https://doi.org/10.1016/j. ijnurstu.2012.07.001

76. Biksegn A, Kenfe T, Matiwos S, Eshetu G. Burnout status at work among health care professionals in a tertiary hospital. Ethiop J Health Sci. 2016;26(2):101-108. https://doi.org/10.4314/ejhs.v26i2.3

77. Shirom A, Nirel N, Vinokur AD. Overload, autonomy, and burnout as predictors of physicians' quality of care. American Psychological Association. Published 2006. https://doi.apa.org/doiLanding?doi=10.1037\%2F1076-8998.11.4.328

78. Coyle D, Edwards D, Hannigan B, Fothergill A, Burnard P. A systematic review of stress among mental health social workers. Int Soc Work. 2005;48(2):201-211. https://doi.org/10.1177/0020872805050492

79. Madathil R, Heck NC, Schuldberg D. Burnout in psychiatric nursing: Examining the interplay of autonomy, leadership style, and depressive symptoms. Arch Psychiatr Nurs. 2014;28(3):160-166. https://doi.org/10.1016/j.apnu.2014.01.002

80. Kowalski C, Ommen O, Driller E, et al. Burnout in nurses - the relationship between social capital in hospitals and emotional exhaustion. J Clin Nurs. 2010;19(1112):1654-1663. https://doi.org/10.1111/j.1365-2702.2009.02989.x

81. Laschinger HKS, Shamian J, Thomson D. Impact of magnet hospital characteristics on nurses' perceptions of trust burnout quality of care, and work satisfaction. Nurs Econ. 2001;19(5):209-209. https://go.gale.com/ps/i. do? $p=A O N E \& s w=w \& i s s n=07461739 \& v=2.1 \& i t=r \& i d=G A L E \% 7 C A 79196525 \&$ sid

82. Moloney W, Boxall P, Parsons M, Cheung G. Factors predicting Registered Nurses' intentions to leave their organization and profession: $A$ job demands-resources framework. J Adv Nurs. 2018;74(4):864-875. https://doi.org/10.1111/jan.13497

83. Bandura A. Self-efficacy. I: V. S. Ramachandran (red.), Encyclopedia of human behavior (vol. s. ). New York: Academic Press. undefined. Published online 1994. https://www.semanticscholar.org/paper/Self-efficacy.-I\%3A-V.-S.-Ramachandran(red.)\%2C-of-s.-Bandura/00dee52a4d1d98e48ed052af174e2e8bcad71563 
84. Mata ÁN de S, de Azevedo KPM, Braga LP, et al. Training in communication skills for self-efficacy of health professionals: A systematic review. Hum Resour Health. 2021;19(1):30. https://doi.org/10.1186/s12960-021-00574-3

85. Maunder RG, Lancee WJ, Mae R, et al. Computer-assisted resilience training to prepare healthcare workers for pandemic influenza: A randomized trial of the optimal dose of training. BMC Health Serv Res. 2010;10(1):72. https://doi. org/10.1186/1472-6963-10-72

86. Shoji K, Cieslak R, Smoktunowicz E, Rogala A, Benight CC, Luszczynska A. Associations between job burnout and self-efficacy: A meta-analysis. Anxiety Stress Coping. 2016;29(4):367-386. https://doi.org/10.1080/10615806.2015.105 8369

87. Milam LA, Cohen GL, Mueller C, Salles A. The relationship between self-efficacy and well-being among surgical residents. J Surg Educ. 2019;76(2):321-328. https:// doi.org/10.1016/j.jsurg.2018.07.028

88. Ong J, Swift $C$, Bath $M$, et al. The prevalence of burnout, risk factors, and jobrelated stressors in gastroenterologists: A systematic review. J Gastroenterol Hepatol. 2021;36(9):2338-2348. https://doi.org/10.1111/jgh.15488

89. Purvanova RK, Muros JP. Gender differences in burnout: A meta-analysis. J Vocat Behav. 2010;77(2):168-185. https://doi.org/10.1016/j.jvb.2010.04.006

90. Pulcrano M, Evans SRT, Sosin M. Quality of life and burnout rates across surgical specialties: A systematic review. JAMA Surg. 2016;151(10):970-978. https://doi. org/10.1001/jamasurg.2016.1647

91. Garrosa E, Moreno-Jiménez B, Liang Y, González JL. The relationship between socio-demographic variables, job stressors, burnout, and hardy personality in nurses: An exploratory study. Int J Nurs Stud. 2008;45(3):418-427. https://doi. org/10.1016/j.ijnurstu.2006.09.003

92. Canadian Medical Association. CMA National Physician Health Survey: A National Snapshot.; 2018:32. https://www.cma.ca/sites/default/files/2018-11/nphsurvey-e.pdf

93. Maunder RG, Leszcz M, Savage D, et al. Applying the lessons of SARS to pandemic influenza. Can J Public Health. 2008;99(6):486-488. https://doi.org/10.1007/ BF03403782

94. Winwood PC, Colon R, McEwen K. A practical measure of workplace resilience: Developing the resilience at work scale. J Occup Environ Med. 2013;55(10):12051212. https://doi.org/10.1097/JOM.0b013e3182a2a60a

95. Sinsky CA, Biddison LD, Mallick A, et al. Organizational evidence-based and promising practices for improving clinician well-being. NAM Perspect. Published online November 2, 2020. https://doi.org/10.31478/202011a

96. Cañadas-De la Fuente GA, Vargas C, San Luis C, García I, Cañadas GR, De la Fuente El. Risk factors and prevalence of burnout syndrome in the nursing profession. Int J Nurs Stud. 2015;52(1):240-249. https://doi.org/10.1016/j.ijnurstu.2014.07.001

97. van der Wal RAB, Bucx MJL, Hendriks JCM, Scheffer G-J, Prins JB. Psychological distress, burnout and personality traits in Dutch anaesthesiologists: A survey. Eur J Anaesthesiol EJA. 2016;33(3):179-186. https://doi.org/10.1097/ EJA.0000000000000375

98. Sun J-W, Lin P-Z, Zhang H-H, Li J-H, Cao F-L. A non-linear relationship between the cumulative exposure to occupational stressors and nurses' burnout and the potentially emotion regulation factors. J Ment Health. 2018;27(5):409-415. 
https://doi.org/10.1080/09638237.2017.1385740

99. Bruyneel A, Smith P, Tack J, Pirson M. Prevalence of burnout risk and factors associated with burnout risk among ICU nurses during the COVID-19 outbreak in French speaking Belgium. Intensive Crit Care Nurs. 2021;65:103059. https://doi. org/10.1016/j.iccn.2021.103059

100. Giusti EM, Pedroli E, D'Aniello GE, et al. The psychological impact of the COVID-19 outbreak on health professionals: A cross-sectional study. Front Psychol. 2020;11:1684. https://doi.org/10.3389/fpsyg.2020.01684

101. Naldi A, Vallelonga F, Liberto AD, et al. COVID-19 pandemic-related anxiety, distress and burnout: prevalence and associated factors in healthcare workers of North-West Italy. BJPsych Open. 2021;7(1). https://doi.org/10.1192/bjo.2020.161

102. de Wit K, Mercuri M, Wallner C, et al. Canadian emergency physician psychological distress and burnout during the first 10 weeks of COVID-19: A mixed-methods study. J Am Coll Emerg Physicians Open. 2020;1(5):1030-1038. https://doi.org/10.1002/emp2.12225

103. Kok N, van Gurp J, Teerenstra S, et al. Coronavirus disease 2019 immediately increases burnout symptoms in ICU professionals: A longitudinal cohort study. Crit Care Med.2021;49(3):419-427.https://doi.org/10.1097/CCM.0000000000004865

104. Khan N, Palepu A, Dodek P, et al. Cross-sectional survey on physician burnout during the COVID-19 pandemic in Vancouver, Canada: The role of gender, ethnicity and sexual orientation. BMJ Open. 2021;11(5):e050380. https://doi.org/10.1136/ bmjopen-2021-050380

105. Maunder RG, Heeney ND, Kiss A, et al. Psychological impact of the COVID-19 pandemic on hospital workers over time: Relationship to occupational role, living with children and elders, and modifiable factors. Gen Hosp Psychiatry. 2021;71:8894. https://doi.org/10.1016/j.genhosppsych.2021.04.012

106. Hu D, Kong Y, Li W, et al. Frontline nurses' burnout, anxiety, depression, and fear statuses and their associated factors during the COVID-19 outbreak in Wuhan, China: A large-scale cross-sectional study. EClinicalMedicine. 2020;24. d https:// doi.org/10.1016/j.eclinm.2020.100424

107. Lasalvia A, Amaddeo F, Porru S, et al. Levels of burn-out among healthcare workers during the COVID-19 pandemic and their associated factors: A crosssectional study in a tertiary hospital of a highly burdened area of north-east Italy. BMJ Open. 2021;11(1):e045127. https://doi.org/10.1136/bmjopen-2020-045127

108. Lin Y-Y, Pan Y-A, Hsieh Y-L, et al. COVID-19 pandemic is associated with an adverse impact on burnout and mood disorder in healthcare professionals. Int J Environ Res Public Health. 2021;18(7):3654. https://doi.org/10.3390/ ijerph18073654

109. Nishimura Y, Miyoshi T, Hagiya H, Kosaki Y, Otsuka F. Burnout of healthcare workers amid the COVID-19 pandemic: A Japanese cross-sectional survey. Int J Environ Res Public Health. 2021;18(5):2434. https://doi.org/10.3390/ ijerph18052434

110. Pniak B, Leszczak J, Adamczyk M, Rusek W, Matłosz P, Guzik A. Occupational burnout among active physiotherapists working in clinical hospitals during the COVID-19 pandemic in south-eastern Poland. Work. 2021;68(2):285-295. https:// doi.org/10.3233/WOR-203375

111. Chen R, Sun C, Chen J-J, et al. A large-scale survey on trauma, burnout, and posttraumatic growth among nurses during the COVID-19 pandemic. Int J Ment Health Nurs. 2021;30(1):102-116. https://doi.org/10.1111/inm.12796 
112. Galanis P, Vraka I, Fragkou D, Bilali A, Kaitelidou D. Nurses' burnout and associated risk factors during the COVID-19 pandemic: A systematic review and meta-analysis. J Adv Nurs. 2021;77(8):3286-3302. https://doi.org/10.1111/ jan.14839

113. Lou NM, Montreuil T, Feldman LS, et al. Nurses' and physicians' distress, burnout, and coping strategies during COVID-19: Stress and impact on perceived performance and intentions to quit. J Contin Educ Health Prof. Published online October 1, 2021. https://doi.org/10.1097/CEH.0000000000000365

114. Jalili M, Niroomand M, Hadavand F, Zeinali K, Fotouhi A. Burnout among healthcare professionals during COVID-19 pandemic: A cross-sectional study. Int Arch Occup Environ Health. 2021;94(6):1345-1352. https://doi.org/10.1007/ s00420-021-01695-x

115. Matsuo T, Kobayashi D, Taki F, et al. Prevalence of health care worker burnout during the Coronavirus disease 2019 (COVID-19) pandemic in Japan. JAMA Netw Open. 2020;3(8):e2017271. https://doi.org/10.1001/ jamanetworkopen.2020.17271

116. Sagherian K, Steege LM, Cobb SJ, Cho H. Insomnia, fatigue and psychosocial well-being during COVID-19 pandemic: A cross-sectional survey of hospital nursing staff in the United States. J Clin Nurs. https://doi.org/10.1111/jocn.15566

117. Abdelhafiz AS, Ali A, Ziady HH, Maaly AM, Alorabi M, Sultan EA. Prevalence, associated factors, and consequences of burnout among Egyptian physicians during COVID-19 pandemic. Front Public Health. 2020;8:864. https://doi. org/10.3389/fpubh.2020.590190

118. Ismail TI, Shehata SF, Mahrous RSS. Occupational stress and burnout among frontline Egyptian anesthesiologists during COVID-19 outbreak in Egypt. Egypt J Anaesth. 2021;37(1):91-99. https://doi.org/10.1080/11101849.2021.1891704

119. Pereira JM, Silva C, Freitas D, Salgado A. Burnout among Portuguese radiographers during the COVID-19 pandemic. Radiography. Published online May 18, 2021. https://doi.org/10.1016/j.radi.2021.05.001

120. Orrù G, Marzetti F, Conversano $C$, et al. Secondary traumatic stress and burnout in healthcare workers during COVID-19 outbreak. Int J Environ Res Public Health. 2021;18(1):337. https://doi.org/10.3390/ijerph18010337

121. Aydin Sayilan A, Kulakaç N, Uzun S. Burnout levels and sleep quality of COVID-19 heroes. Perspect Psychiatr Care. 2021;57(3):1231-1236. https://doi. org/10.1111/ppc.12678

122. Johnston K, O'Reilly CL, Scholz B, Georgousopoulou EN, Mitchell I. Burnout and the challenges facing pharmacists during COVID-19: Results of a national survey. Int J Clin Pharm. 2021;43(3):716-725. https://doi.org/10.1007/s11096021-01268-5

123. Afonso AM, Cadwell JB, Staffa SJ, Zurakowski D, Vinson AE. Burnout rate and risk factors among anesthesiologists in the United States. Anesthesiology. 2021;134(5):683-696. https://doi.org/10.1097/ALN.0000000000003722

124. Prasad K, McLoughlin C, Stillman M, et al. Prevalence and correlates of stress and burnout among U.S. healthcare workers during the COVID-19 pandemic: A national cross-sectional survey study. EClinicalMedicine. 2021;35. https://doi. org/10.1016/j.eclinm.2021.100879

125. Ruotsalainen JH, Verbeek JH, Mariné A, Serra C. Preventing occupational stress in healthcare workers. Cochrane Database Syst Rev. 2015;(4). https://doi. org/10.1002/14651858.CD002892.pub5 
126. Zhang $X$, Song $Y$, Jiang $T$, Ding $N$, Shi T. Interventions to reduce burnout of physicians and nurses: An overview of systematic reviews and metaanalyses. Medicine (Baltimore). 2020;99(26):e20992. https://doi.org/10.1097/ MD.0000000000020992

127. Panagioti M, Panagopoulou E, Bower $\mathrm{P}$, et al. Controlled interventions to reduce burnout in physicians: A systematic review and meta-analysis. JAMA Intern Med. 2017;177(2):195-205. https://doi.org/10.1001/jamainternmed.2016.7674

128. Price D, Carr RS, Howard M. Caring for the carers. Can Fam Physician. 2013;59(11):1145-1147. https://www.ncbi.nlm.nih.gov/pmc/articles/ PMC3828084/

129. Shanafelt TD, Mungo M, Schmitgen J, et al. Longitudinal study evaluating the association between physician burnout and changes in professional work effort. Mayo Clin Proc. 2016;91(4):422-431. https://doi.org/10.1016/j. mayocp.2016.02.001

130. Shanafelt TD, Balch CM, Dyrbye L, et al. Special report: Suicidal ideation among American surgeons. Arch Surg. 2011;146(1):54-62. https://doi.org/10.1001/ archsurg.2010.292

131. AlJhani S, AlHarbi H, AlJameli S, Hameed L, AlAql K, Alsulaimi M. Burnout and coping among healthcare providers working in Saudi Arabia during the COVID-19 pandemic. Middle East Curr Psychiatry. 2021;28(1):29. https://doi.org/10.1186/ s43045-021-00108-6

132. Amanullah S, Ramesh Shankar R. The Impact of COVID-19 on physician burnout globally: A review. Healthcare. 2020;8(4):421. https://doi.org/10.3390/ healthcare8040421

133. Cotel A, Golu F, Pantea Stoian A, et al. Predictors of burnout in healthcare workers during the COVID-19 pandemic. Healthcare. 2021;9(3):304. https://doi. org/10.3390/healthcare9030304

134. Hesselink G, Straten L, Gallée L, et al. Holding the frontline: A cross-sectional survey of emergency department staff well-being and psychological distress in the course of the COVID-19 outbreak. BMC Health Serv Res. 2021;21(1):525. https:// doi.org/10.1186/s12913-021-06555-5

135. Lamb D, Gnanapragasam S, Greenberg N, et al. Psychosocial impact of the COVID-19 pandemic on 4378 UK healthcare workers and ancillary staff: Initial baseline data from a cohort study collected during the first wave of the pandemic. Occup Environ Med. Published online June 27, 2021. https://doi.org/10.1136/ oemed-2020-107276

136. Ness MM, Saylor J, DiFusco LA, Evans K. Leadership, professional quality of life and moral distress during COVID-19: A mixed-methods approach. J Nurs Manag. https://doi.org/10.1111/jonm.13421

137. Silverman HJ, Kheirbek RE, Moscou-Jackson G, Day J. Moral distress in nurses caring for patients with Covid-19. Nurs Ethics. Published online April 29, 2021:09697330211003217. https://doi.org/10.1177/09697330211003217

138. McAndrew NS, Leske J, Schroeter K. Moral distress in critical care nursing: The state of the science. Nurs Ethics. 2018;25(5):552-570. https://doi. org/10.1177/0969733016664975

139. Roslan NS, Yusoff MSB, Asrenee AR, Morgan K. Burnout prevalence and Its associated factors among Malaysian healthcare workers during COVID-19 pandemic: An embedded mixed-method study. Healthcare. 2021;9(1):90. https:// doi.org/10.3390/healthcare9010090 
140. Maunder RG, Lancee WJ, Balderson KE, et al. Long-term psychological and occupational effects of providing hospital healthcare during SARS outbreak. Emerg Infect Dis J - CDC. 2006;12. https://doi.org/10.3201/eid1212.060584

141. Registered Nurses' Association of Ontario (RNAO). Work and wellbeing survey results.; 2021. https://rnao.ca/sites/rnao-ca/files/Nurses_Wellbeing_Survey_ Results_-_March_31.pdf

142. Grant K. Canadian nurses are leaving in droves, worn down by 16 merciless months on the front lines of COVID-19. The Globe and Mail. Published July 16, 2021. https://www.theglobeandmail.com/canada/article-canadian-nurses-areleaving-in-droves-worn-down-by-16-merciless-months/

143. Gorman M. Nova Scotia Health to close some surgery beds, redeploy nurses to areas with critical needs. CBC. Published August 25, 2021. https://www.cbc. $\mathrm{ca} /$ news/canada/nova-scotia/health-care-nurses-emergency-departmentstaffing-1.6151994

144. Hunt S, Producer Ctvn ca D, Contact F|. Elective surgeries cancelled in Calgary, Fort Macleod emergency room closed as COVID-19 surge continues. CTV News. Published September 8, 2021. https://calgary.ctvnews.ca/elective-surgeriescancelled-in-calgary-fort-macleod-emergency-room-closed-as-covid-19-surgecontinues-1.5578056

145. Stevenson V. Another Quebec ER had to shut down because of a lack of nurses, who are exhausted. CBC. Published September 16, 2021. https://www. cbc.ca/news/canada/montreal/suroit-hospital-er-closes-overnight-1.6178786

146. Cox A. Staff shortages at 2 N.B. hospitals lead to closures of labour, obstetrics units. CBC. Published August 19, 2021. https://www.cbc.ca/news/canada/newbrunswick/hospital-labour-obstetrics-new-brunswick-1.6146501

147. Payne E. Signing bonuses are a sign of the times as desperate hospitals seek nurses. ottawacitizen. Published June 11, 2021. https://ottawacitizen.com/news/ local-news/signing-bonuses-are-a-sign-of-the-times-as-desperate-hospitals-seeknurses

148. Research, Analysis, and Evaluation Branch. Evidence synthesis briefing note: Best practices for supporting health care worker burnout following intense professional commitment. COVID-19 Evidence Synthesis Network; 2021. https:// esnetwork.ca/wp-content/uploads/2021/05/61.-BN-on-Health-Care-WorkerBurnout_17-June-21_v1.pdf

149. Moat KA, Waddell K, Lavis JN. Planning for the future health workforce of Ontario.; 2016:60. https://www.mcmasterforum.org/docs/default-source/ product-documents/evidence-briefs/workforce-planning-eb.pdf

150. Goh Y-S, Lee A, Chan SW-C, Chan MF. Profiling nurses' job satisfaction, acculturation, work environment, stress, cultural values and coping abilities: A cluster analysis. Int J Nurs Pract. 2015;21(4):443-452. https://doi.org/10.1111/ ijn.12318

151. Labrague $L \mathrm{j} .$, McEnroe-Petitte $\mathrm{D} \mathrm{m}$. Job stress in new nurses during the transition period: An integrative review. Int Nurs Rev. 2018;65(4):491-504. https:// doi.org/10.1111/inr.12425

152. Zhang Y, Wu J, Fang Z, Zhang Y, Wong FKY. Newly graduated nurses' intention to leave in their first year of practice in Shanghai: A longitudinal study. Nurs Outlook. 2017;65(2):202-211. https://doi.org/10.1016/j.outlook.2016.10.007

153. Brook J, Aitken L, Webb R, MacLaren J, Salmon D. Characteristics of successful interventions to reduce turnover and increase retention of early career nurses: 
A systematic review. Int J Nurs Stud. 2019;91:47-59. https://doi.org/10.1016/j. ijnurstu.2018.11.003

154. Scott JG, Sochalski J, Aiken L. Review of magnet hospital research: Findings and implications for professional nursing practice. JONA J Nurs Adm. 1999;29(1):919. https://journals.Iww.com/jonajournal/Abstract/1999/01000/Review_of_ Magnet_Hospital_Research_Findings_and.3.aspx

155. Kelly LA, McHugh MD, Aiken LH. Nurse outcomes in magnet and non-magnet hospitals. JONA J Nurs Adm. 2012;42(10):S44. https://doi.org/10.1097/01. NNA.0000420394.18284.4f

156. McHugh MD, Kelly LA, Smith HL, Wu ES, Vanak JM, Aiken LH. Lower mortality in magnet hospitals. JONA J Nurs Adm. 2013;43(10):S4. https://doi.org/10.1097/01. NNA.0000435145.39337.d5

157. Jeffs L, Merkley J, Canzian S, Taggart R, Harris IA and A. Balancing resiliency and new accountabilities: Insights from chief nurse executives amid the COVID-19 pandemic. Nurs Leadersh. 2020;33(4). https://www.longwoods. com/content/26425/nursing-leadership/balancing-resiliency-and-newaccountabilities-insights-from-chief-nurse-executives-amid-the-covid-1 